TITLE:

\title{
Microbial production of conjugated fatty acids
}

AUTHOR(S):

Kishino, Shigenobu; Ogawa, Jun; Yokozeki, Kenzo; Shimizu, Sakayu

\section{CITATION:}

Kishino, Shigenobu ...[et al]. Microbial production of conjugated fatty acids. Lipid Technology 2009, 21(8-9): 177-181

ISSUE DATE:

2009-09

URL:

http://hdl.handle.net/2433/91449

\section{RIGHT:}

c 2009 WILEY-VCH Verlag GmbH \& Co. KGaA, Weinheim.; この論文は出 版社版でありません。引用の際には出版社版をご確認ご利用ください - ; This is not the published version. Please cite only the published version. 


\section{Microbial production of conjugated fatty acids}

\section{Shigenobu Kishino, Jun Ogawa, Kenzo Yokozeki and Sakayu Shimizu}

5 Shigenobu Kishino is assistant professor at the Laboratory of Industrial Microbiology and the Laboratory of Fermentation Physiology and Applied Microbiology, Division of Applied Life Sciences, Graduate School of Agriculture, Kyoto University, Kitashirakawa-oiwakecho, Sakyo-ku, Kyoto 606-8502, Japan; tel.: +81-75-753-6114; fax: +81-75-753-6128; e-mail: kishino@kais.kyoto-u.ac.jp

10 Jun Ogawa is professor at Laboratory of Fermentation Physiology and Applied Microbiology, Division of Applied Life Sciences, Graduate School of Agriculture and the Research Division of Microbial Sciences, Kyoto University, Sakyo-ku, Kyoto 606-8502, Japan; Tel.: +81-75-753-6122 fax: +81-75-753-6128; e-mail: ogawa@kais.kyoto-u.ac.jp Kenzo Yokozeki is professor at the Laboratory of Industrial Microbiology, Graduate

15 School of Agriculture, Kyoto University, Kitashirakawa-oiwakecho, Sakyo-ku, Kyoto 606-8502, Japan; tel.: +81-75-753-6113; fax: +81-75-753-6128; e-mail: kenzo@kais.kyoto-u.ac.jp

Sakayu Shimizu is professor at the Laboratory of Fermentation Physiology and Applied Microbiology, Division of Applied Life Sciences, Graduate School of Agriculture, Kyoto

20 University, Kitashirakawa-oiwakecho, Sakyo-ku, Kyoto 606-8502, Japan; tel.: +81-75-753-6115; fax: +81-75-753-6128; e-mail: sim@kais.kyoto-u.ac.jp

\section{Summary}

Conjugated fatty acids have attracted much attention as a novel type of biologically beneficial functional lipid. Some isomers of conjugated linoleic acid (CLA) reduce carcinogenesis, atherosclerosis and body fat. Considering the use of CLA for medicinal and nutraceutical purposes, a safe isomer-selective process of production is required. The introduction of biological reactions for CLA production could be an answer. We screened microbial reactions useful for CLA production and found several unique reactions in microorganisms. Lactic acid bacteria produced CLA from linoleic acid. The 
CLA, which was obtained as the free fatty acid form, comprised a mixture of cis-9,trans-11-octadecadienoic acid (18:2) and trans-9,trans-11-18:2. Furthermore, lactic acid bacteria transformed ricinoleic acid [12-hydroxy-cis-9-octadecenoic acid (18:1)] to CLA (a mixture of cis-9,trans-11-18:2 and trans-9,trans-11-18:2). Castor oil,

5 rich in the triacylglycerol form of ricinoleic acid, was also found to act as a substrate for CLA production by lactic acid bacteria with the aid of lipase-catalyzed triacylglycerol hydrolysis. Filamentous fungi transformed trans-vaccenic acid (trans-11-18:1) to cis-9,trans-11-18:2 by 49 desaturation. This CLA was obtained as a triacylglycerol.

10 In addition, lactic acid bacteria produced conjugated trienoic fatty acids from $\alpha$ - and $\gamma$-linolenic acid. The trienoic fatty acids produced from $\alpha$-linolenic acid were cis-9,trans-11,cis-15-octadecatrienoic acid (18:3) and trans-9,trans-11,cis-15-18:3. Those produced from $\gamma$-linolenic acid were cis-6,cis-9,trans-11-18:3 and cis-6,trans-9,trans-11-18:3.

\section{Introduction}

Various fatty acids with conjugated double bonds occur in nature. For example, edible fats derived from ruminant animals contain conjugated linoleic acid (CLA), which mainly consists of cis-9,trans-11- and trans-10,cis-12-octadecadienoic acid (18:2). The occurrence of conjugated fatty acids has also been reported in plants, for example, $\alpha$-eleostearic acid [cis-9,trans-11,trans-13-octadecatrienoic acid (18:3)] in Momordica charantia seed oil. The secondary metabolism of fatty acids by marine algae involves polyunsaturated fatty acids containing conjugated olefin 
systems, for example, cis-5,trans-7,trans-9,cis-14-eicosatetraenoic acid (20:4) produced from arachidonic acid (cis-5,cis-8,cis-11,cis-14-20:4). These conjugated fatty acids have attracted much attention as a novel type of biologically beneficial functional lipid. In particular, the unique activities

5 of CLA have been intensively studied, and CLA is expected to be a potential material for pharmaceuticals and dietary supplements. CLA reduces carcinogenesis, atherosclerosis, and body fat.

Today CLA, as a dietary supplement, is produced through chemical isomerization of linoleic acid, which results in the coproduction of CLA isomers. However, recent studies have revealed that each isomer can have different effects on metabolism and cell functions and acts through different cell signaling pathways. To date, cis-9,trans-11 and trans-10,cis-12 isomers have been paid particular attention because of their remarkable biological activities. Considering the use of CLA for medicinal

15 and nutraceutical purposes, a safe isomer-selective process is required. A bioprocess is of potential use for this purpose and we review microbial production of conjugated fatty acids in this paper.

\section{Useful reaction for CLA production by microorganisms}

\section{Isomerization of linoleic acid to CLA}

To establish efficient processes for CLA production, we screened lactic acid bacteria for the ability to produce CLA from linoleic acid. More than 250 bacterial strains from 14 genera were examined, and strains belonging 
to the genera Enterococcus, Pediococcus, Propionibacterium, and Lactobacillus were found to produce considerable amounts of CLA from linoleic acid. All strains produced two specific isomers of CLA, i.e., cis-9,trans-11-18:2 (CLA1) and trans-9,trans-11-18:2 (CLA2), together

5 with two hydroxy fatty acid, i.e., 10-hydroxy-trans-12-octadecenoic acid (18:1) (HY1) and 10-hydroxy-cis-12-18:1 (HY2). From these strains, Lactobacillus plantarum AKU 1009a was selected for its potential to produce CLA from linoleic acid ${ }^{1)}$. The mechanism of CLA production from linoleic acid in relation to hydroxy fatty acid production was investigated using L. plantarum AKU 1009a as a representative strain, and we showed that linoleic acid isomerization to CLA by lactic acid bacteria consists of at least two successive reactions, i.e., the hydration of linoleic acid to 10-hydroxy-18:1 and the dehydrating isomerization of the hydroxy fatty acid to CLA (Fig. 1). Only the free form of linoleic acid acted as a substrate

15 for CLA production by lactic acid bacteria, i.e., the ester and triacylglycerol of linoleic acid did not. Washed cells of L. plantarum exhibiting a high level of CLA production were obtained by cultivation in nutrient medium containing free linoleic acid $[0.06 \%(\mathrm{w} / \mathrm{v})]$. The CLA-producing reaction using the washed cells as a catalyst proceeded well even under aerobic conditions with free linoleic acid mixed with bovine serum albumin as the substrate. Under the optimum reaction conditions, the washed cells of $L$. plantarum [33\% (wet w/v)] produced $40 \mathrm{mg} / \mathrm{ml}$ CLA from $120 \mathrm{mg} / \mathrm{ml}$ of linoleic acid in $108 \mathrm{~h}$. The resulting CLA comprised a mixture of CLA1 (38\% of total CLA) and CLA2 (62\% of total CLA), and accounted for 50\% 
of the total fatty acids obtained. A higher yield (80\% molar yield from linoleic acid) was attained using the washed cells of L. plantarum [23\% (wet w/v)] and $26 \mathrm{mg} / \mathrm{ml}$ of linoleic acid in $96 \mathrm{~h}$, resulting in CLA production of $20 \mathrm{mg} / \mathrm{ml}$ [consisting of CLA1 (2\%) and CLA2 (98\%), and

5 accounting for $80 \%$ of the total fatty acids obtained]. Most of the CLA produced was accumulated as intracellular or cell-associated lipids in the free form; thus, it was simple to recover CLA by centrifugation, and the cells themselves could be used as a source of CLA.

$10 \quad$ ((Figure 1))

\section{Dehydration of ricinoleic acid to CLA}

The transformation of hydroxy fatty acids by lactic acid bacteria was investigated using Lactobacillus plantarum AKU 1009a as a representative

15 strain. Among the various hydroxy fatty acids examined, this strain transformed ricinoleic acid (12-hydroxy-cis-9-18:1) into CLA (a mixture of CLA1 and CLA2). The ability to produce CLA from ricinoleic acid was found to be widely distributed in lactic acid bacteria. There are two possible pathways for CLA synthesis from ricinoleic acid by lactic acid bacteria: (i) direct transformation of ricinoleic acid into CLA through dehydration at the $\Delta 11$ position, and (ii) three-step transformation via linoleic acid through dehydration at the $\Delta 12$ position and successive isomerization of linoleic acid (Fig. 1). Only the free form of ricinoleic acid acted as a substrate for CLA production by lactic acid bacteria, i.e., the 
ester and triacylglycerol of ricinoleic acid did not.

More than 250 bacterial strains from 14 genera were examined, and strains belonging to the genera Streptococcus, Leuconostoc, Pediococcus, Propionibacterium, and Lactobacillus were found to produce considerable

5 amounts of CLA from ricinoleic acid. From these strains, L. plantarum JCM 1551 was selected for its potential to produce CLA from ricinoleic $\operatorname{acid}^{2)}$. This strain had the highest CLA-producing activity when it was cultivated in medium supplemented with $0.2 \%(\mathrm{w} / \mathrm{v})$ of a mixture of $\alpha$-linolenic acid and linoleic acid in the ratio of 1:5. The CLA-producing 10 reaction using the washed cells as a catalyst proceeded well under micro-aerobic conditions with free ricinoleic acid mixed with bovine serum albumin as the substrate. Under the optimum reaction conditions using the washed cells of $L$. plantarum [12\% (wet w/v)], $2.4 \mathrm{mg} / \mathrm{ml}$ CLA was produced from $3.4 \mathrm{mg} / \mathrm{ml}$ ricinoleic acid in $90 \mathrm{~h}$. The CLA produced,

15 which was obtained in the free fatty acid form, consisted of CLA1 (21\% of total CLA) and CLA2 (79\% of total CLA), and accounted for $72 \%$ of the total fatty acids obtained. Seventy percent of the CLA produced was accumulated as intracellular or cell-associated lipids, the remainder was found in the reaction supernatant. The unreacted ricinoleic acid was mainly

20 found in the supernatant.

Furthermore, castor oil, which is rich in the triacylglycerol form of ricinoleic acid, was available as a substrate for CLA production by lactic acid bacteria with the aid of lipase-catalyzed triacylglycerol hydrolysis. Under the optimum conditions using the washed cells of $L$. plantarum JCM 
1551 [12\% (wet w/v)] and Lipase M “Amano" 10 as the catalyst, 2.7 $\mathrm{mg} / \mathrm{ml}$ CLA was produced from $5.0 \mathrm{mg} / \mathrm{ml}$ castor oil in $99 \mathrm{~h}$. The CLA produced accounted for $46 \%$ of the total fatty acids obtained, and consisted of CLA1 (26\%) and CLA2 (74\%) ${ }^{3)}$. Seventy and thirty percent of the CLA

5 produced were accumulated intracellularly (or associated with cells) and extracellularly, respectively, mainly as the free form. The unreacted ricinoleic acid was mainly found in the supernatant, mostly as the free form.

10 Desaturation of trans-vaccenic acid to CLA

We screened about 500 fungal strains and yeasts for the ability to produce CLA from trans-vaccenic acid (trans-11-18:1) through $\Delta 9$ desaturation. The ability was widely distributed in filamentous fungi, and Delacroixia coronata IFO 8586 was selected for its potential to produce CLA from

15 trans-vaccenic acid ${ }^{4)}$. This strain efficiently transformed trans-vaccenic acid and its methyl ester added to culture medium. Under optimum culture conditions in nutrient medium containing $33.3 \mathrm{mg} / \mathrm{ml}$ trans-vaccenic acid methyl ester, this strain produced $10.5 \mathrm{mg} / \mathrm{ml}$ CLA in 7 days. The produced CLA, most of which was obtained as triacylglycerol, comprised of CLA1 20 (98\%) and CLA2 (2\%).

CLA produced by lactic acid bacteria is a free fatty acid. However, triacylglycerol-containing CLA is also interesting from physiological and nutritional viewpoints. As a method for the triacylglycerol production, CLA production - using molds that accumulate lipids as triacylglycerol - is 
promising.

\section{Production of conjugated trienoic fatty acids by lactic acid bacteria}

\section{Transformation of polyunsaturated fatty acids by lactic acid bacteria}

We investigated the substrate spectrum of polyunsaturated fatty acid transformation by the washed cells of Lactobacillus plantarum AKU 1009a. Among various polyunsaturated fatty acids examined $\alpha$-linolenic acid (cis-9,cis-12,cis-15-18:3), $\quad \gamma$-linolenic $\quad$ acid $\quad$ (cis-6,cis-9,cis-12-18:3),

10 pinolenic acid (cis-5,cis-9,cis-12-18:3), and stearidonic acid [cis-6,cis-9,cis-12,cis-15-octadecatetraenoic acid (18:4)] were transformed (Table 1 ). The fatty acids transformed by the strain were $\mathrm{C}_{18}$ fatty acids sharing a cis-9,cis-12 diene system. Three major fatty acids produced from $\alpha$-linolenic acid, were identified as cis-9,trans-11,cis-15-18:3,

15 trans-9,trans-11,cis-15-18:3, and trans-10,cis-15-18:2. Four major fatty acids produced from $\gamma$-linolenic acid were identified as cis-6,cis-9,trans-11-18:3, cis-6,trans-9,trans-11-18:3, cis-6,trans-10-18:2, and trans-10-18:15). The time course of changes in fatty acid composition during $\alpha$-linolenic acid transformation was studied. In the initial stage of

20 the transformation two conjugated trienoic acids namely cis-9,trans-11,cis-15-18:3 and trans-9,trans-11,cis-15-18:3 were accumulated. As the reaction proceeded, the amount of conjugated trienoic acid gradually decreased, followed by an increase in the amount of the dienoic acid of trans-10,cis-15-18:2. Similar results were obtained for 
$\gamma$-linolenic acid transformation. On the basis of these results, we proposed the pathways of $\alpha$ - and $\gamma$-linolenic acid transformation shown in Fig. 2. $L$. plantarum AKU 1009a transformed the cis-9,cis-12 diene system to the conjugated diene systems of cis-9,trans-11 and trans-9,trans-11. These

5 conjugated dienes were further saturated by this strain to the trans-10 monoene. On the basis of the above results, the three major fatty acids produced from stearidonic acid are surmised to be cis-6,cis-9,trans-11,cis-15-18:4， cis-6,trans-9,trans-11,cis-15-18:4， and cis-6,trans-10,cis-15-18:3, and the three major fatty acids produced from

10 pinolenic acid are surmised to be cis-5,cis-9,trans-11-18:3, cis-5,trans-9,trans-11-18:3, and cis-5,trans-10-18:2.

((Table 1 and Figure 2))

15 Preparative production of conjugated $\alpha$ - and $\gamma$-linolenic acid by lactic acid bacteria

Conjugated $\alpha$-linolenic acid (CALA) was produced by the incubation of $\alpha$-linolenic acid with the washed cells of Lactobacillus plantarum AKU 1009a. Washed cells exhibiting high levels of CALA productivity were obtained by cultivation in nutrient medium supplemented with $0.01 \%(\mathrm{w} / \mathrm{v})$ $\alpha$-linolenic acid. The CALA-producing reaction using the washed cells as a catalyst proceeded well under micro-aerobic conditions with free $\alpha$-linolenic acid mixed with bovine serum albumin as the substrate. Under the optimum reaction conditions using $63 \mathrm{mg} / \mathrm{ml}$ of $\alpha$-linolenic acid, the 
washed cells [33\% (wet w/v)] produced $25 \mathrm{mg} / \mathrm{ml}$ of CALA in $72 \mathrm{~h}$. The produced CALA comprised a mixture of the two isomers, i.e., cis-9,trans-11,cis-15-18:3 (CALA1, 67\% of total CALA) and trans-9,trans-11,cis-15-18:3 (CALA2, 33\% of total CALA), and accounted

5 for $48 \%$ of the total fatty acids obtained. Almost stoichiometric conversion was attained using $12 \mathrm{mg} / \mathrm{ml}$ of $\alpha$-linolenic acid and washed cells [20\% (wet w/v)] in $48 \mathrm{~h}$. The $12 \mathrm{mg} / \mathrm{ml}$ of CALA produced consisted of $43 \%$ CALA1 and 57\% CALA2, and accounted for $66 \%$ of the total fatty acids obtained. Forty and sixty percent of the CALA produced were accumulated intracellularly (or associated with cells) and extracellularly, respectively, mainly as the free acid.

Conjugated $\gamma$-linolenic acid (CGLA) was produced by the incubation of $\gamma$-linolenic acid with the washed cells of L. plantarum AKU 1009a. Washed cells exhibiting high levels of CGLA productivity were obtained by

15 cultivation in nutrient medium supplemented with $0.03 \%$ (w/v) $\alpha$-linolenic acid. The CGLA-producing reaction using the washed cells as a catalyst proceeded well under microaerobic conditions with free $\gamma$-linolenic acid mixed with a detergent, $N$-heptyl- $\beta$-D-thioglucoside, as the substrate. Under the optimum reaction conditions using $13 \mathrm{mg} / \mathrm{ml}$ of $\gamma$-linolenic acid, 20 the washed cells [32\% (wet w/v)] produced $8.8 \mathrm{mg} / \mathrm{ml}$ of CGLA in $27 \mathrm{~h}$. The produced CGLA comprised a mixture of the two isomers, i.e., cis-6,cis-9,trans-11-18:3 (CGLA1, 40\% of total CGLA) and cis-6,trans-9,trans-11-18:3 (CGLA2, 60\% of total CGLA), and accounted for $66 \%$ of the total fatty acids obtained. Seventy and thirty percent of the 
CGLA produced were accumulated intracellularly (or associated with cells) and extracellularly, respectively, mainly as the free acid.

\section{Discussion}

In this review, we have examined practical CLA production and reveal that only two CLA isomers (CLA1 and CLA2) were produced from linoleic acid, ricinoleic acid or castor oil by lactic acid bacteria, suggesting that the biological CLA production processes are more isomer-selective than the

10 chemical ones. However, it is still important to control the isomer production ratio for a more selective isomer synthesis. We investigated the factors affecting the isomer ratio in CLA production from linoleic acid, and found that it could be controlled by changing the reaction conditions. For example, addition of L-serine, glucose, $\mathrm{AgNO}_{3}$, or $\mathrm{NaCl}$ to the reaction

15 mixture reduced the production of CLA2, resulting in selective production of CLA1 (about 75\% selectivity) (6). CLA2 is produced with more than 97\% selectivity, if the reaction is performed for longer using a low linoleic acid concentration. Highly selective production of CLA1 is also possible through filamentous fungi catalyzed $\Delta 9$ desaturation to trans-vaccenic acid.

20 The CLA produced by fungi is mainly in the triacylglycerol form, while that produced by lactic acid bacteria is in the free fatty acid form (Fig. 3).

((Figure 3)) 
Not only CLA but also conjugated trienoic acids were produced by the washed cells of lactic acid bacteria. The isomer selectivity of lactic acid bacteria is advantageous for the trienoic acid transformation, which is hard to control by chemical methods.

5 As described in this review, micro-organisms exhibit unique fatty acid transformation reactions, such as isomerization, dehydration, and desaturation, which are useful for conjugated fatty acid production.

\section{Acknowledgements}

10 This work was partially supported by the Industrial Technology Research Grant Program in 2007 (No. 07A08005a to S.K.) from New Energy and Industrial Technology Development Organization (NEDO) of Japan, Grants-in-Aid for Scientific Research (No. 19780056 to S.K., No. 16688004 to J.O., and No. 13876023 to S.S.) and COE for

15 Microbial-Process Development Pioneering Future Production Systems from the Ministry of Education, Culture, Sports, Science, and Technology, Japan. S.K. was a recipient of a Research Fellowship (No. 01985) from the Japan Society for the Promotion of Science for Young Scientists.

\section{References}

1) Kishino, S. et al. (2002) "Conjugated linoleic acid production from linoleic acid by lactic acid bacteria.” J. Am. Oil Chem. Soc., 79, 159-163

2) Ando, A. et al. (2003) "CLA production from ricinoleic acid by lactic acid bacteria” J. Am. Oil Chem. Soc., 80, 889-894. 
3) Ando, A. et al. (2004) "Conjugated linoleic acid production from castor oil by Lactobacillus plantarum JCM 1551” Enzyme Microb. Technol., 35, 40-45.

4) Ando, A. et al. (2009) "Selective production of cis-9,trans-11 isomer of conjugated linoleic acid from trans-vaccenic acid methyl ester by Delacroixia coronata” J. Appl. Microbial., 106, 1697-1704.

5) Kishino, S. et al. (2009) "Metabolic diversity in biohydrogenation of polyunsaturated fatty acids by lactic acid bacteria involving conjugated fatty acid production” Appl. Microbiol. Biotechnol., 84, 87-97.

6) Kishino, S. et al. (2003) "Structural analysis of conjugated linoleic acid production by Lactobacillus plantarum, and factors affecting isomer production” Biosci. Biotechnol. Biochem., 67, 179-182.

Figure 1. Proposed pathway of linoleic acid, ricinoleic acid, and castor oil transformation to CLA by Lactobacillus plantarum.

Figure 2. Proposed pathway of $\alpha$ - and $\gamma$-linolenic acid transformation by Lactobacillus plantarum.

20 Figure 3. Preparative production of CLA by microorganisms from linoleic acid, ricinoleic acid, castor oil, and trans-vaccenic acid. 
Table 1. Transformation of unsaturated fatty acids by Lactobacillus plantarum AKU 1009a

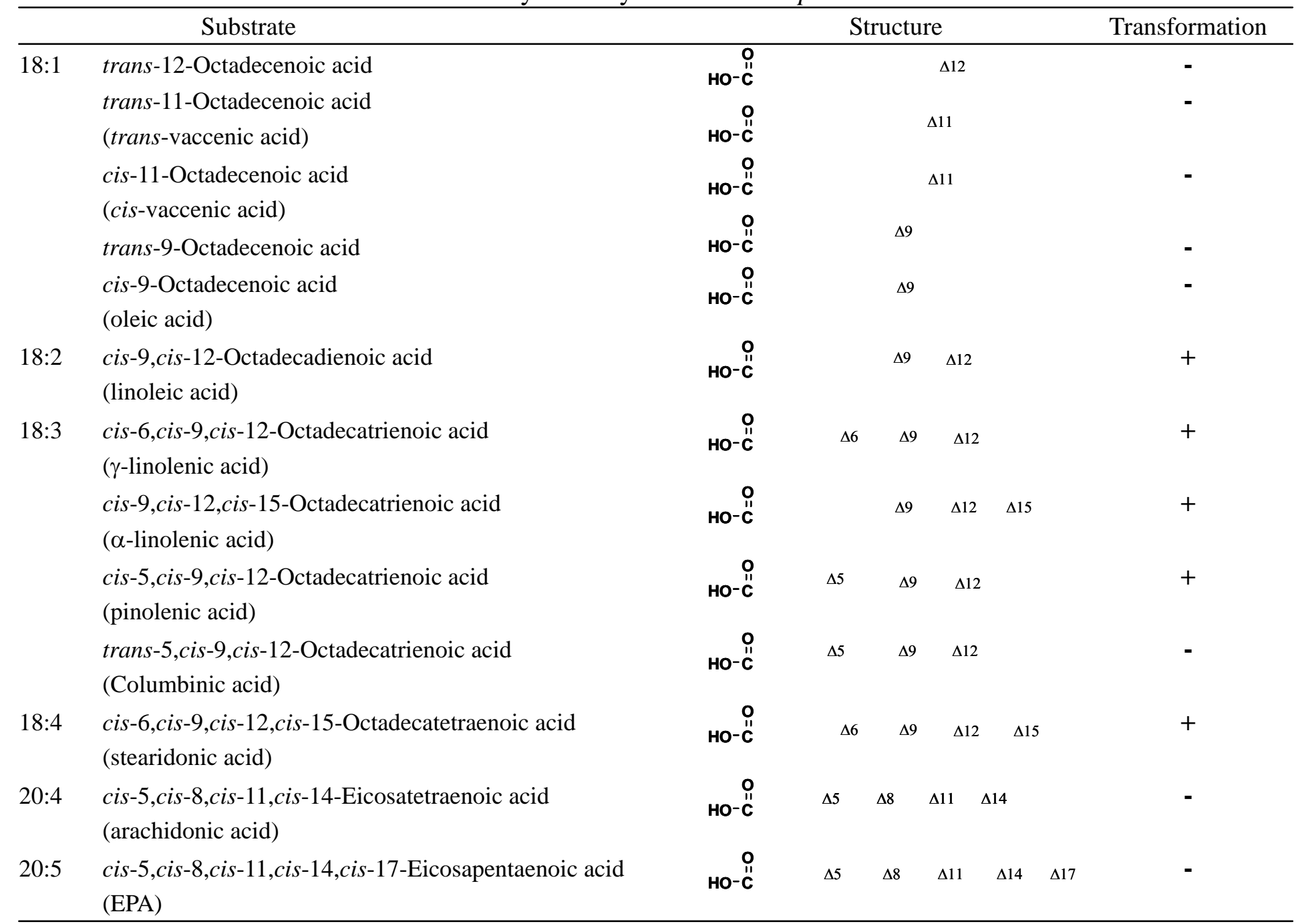



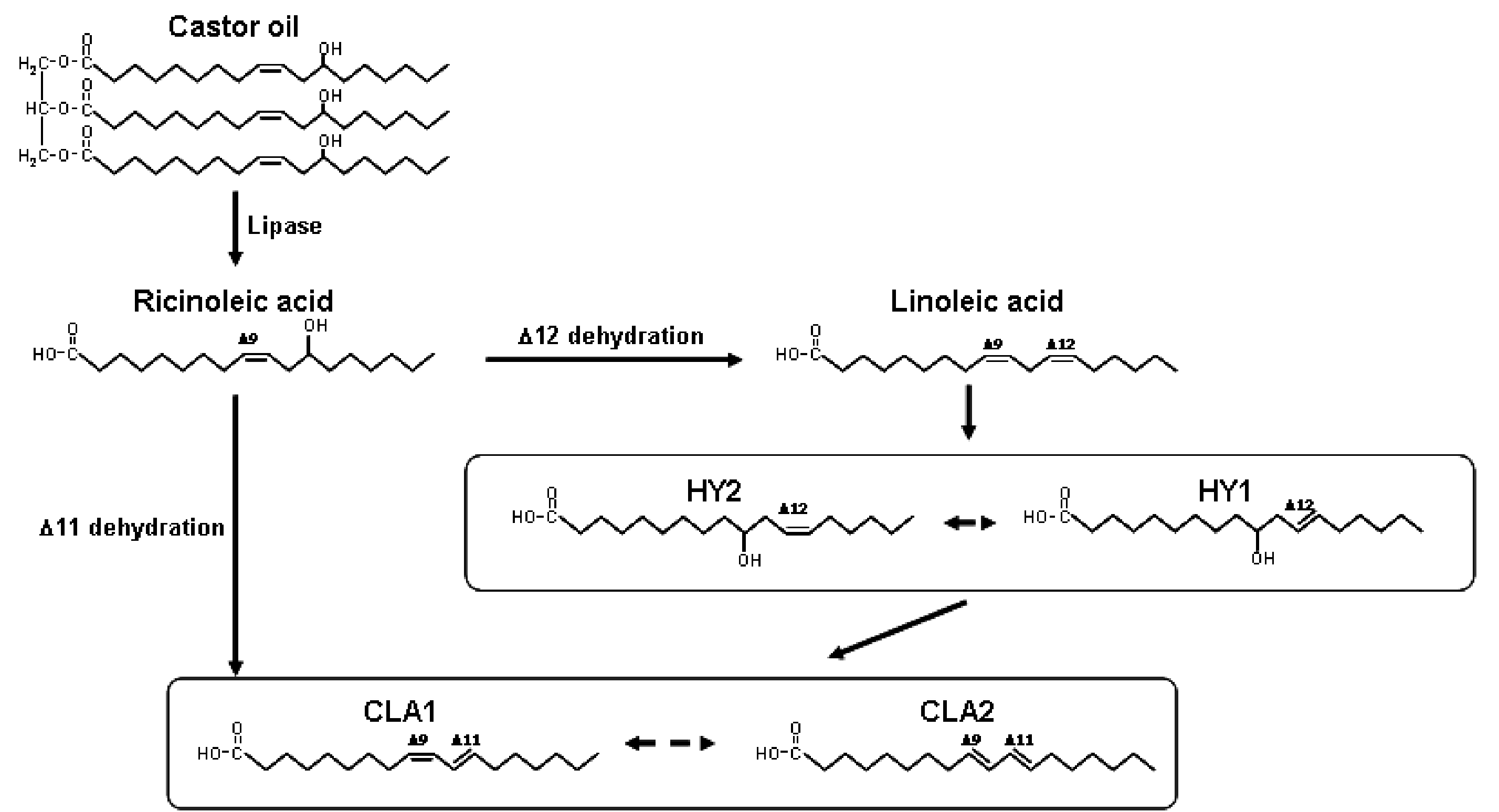
$\alpha$-Linolenic acid

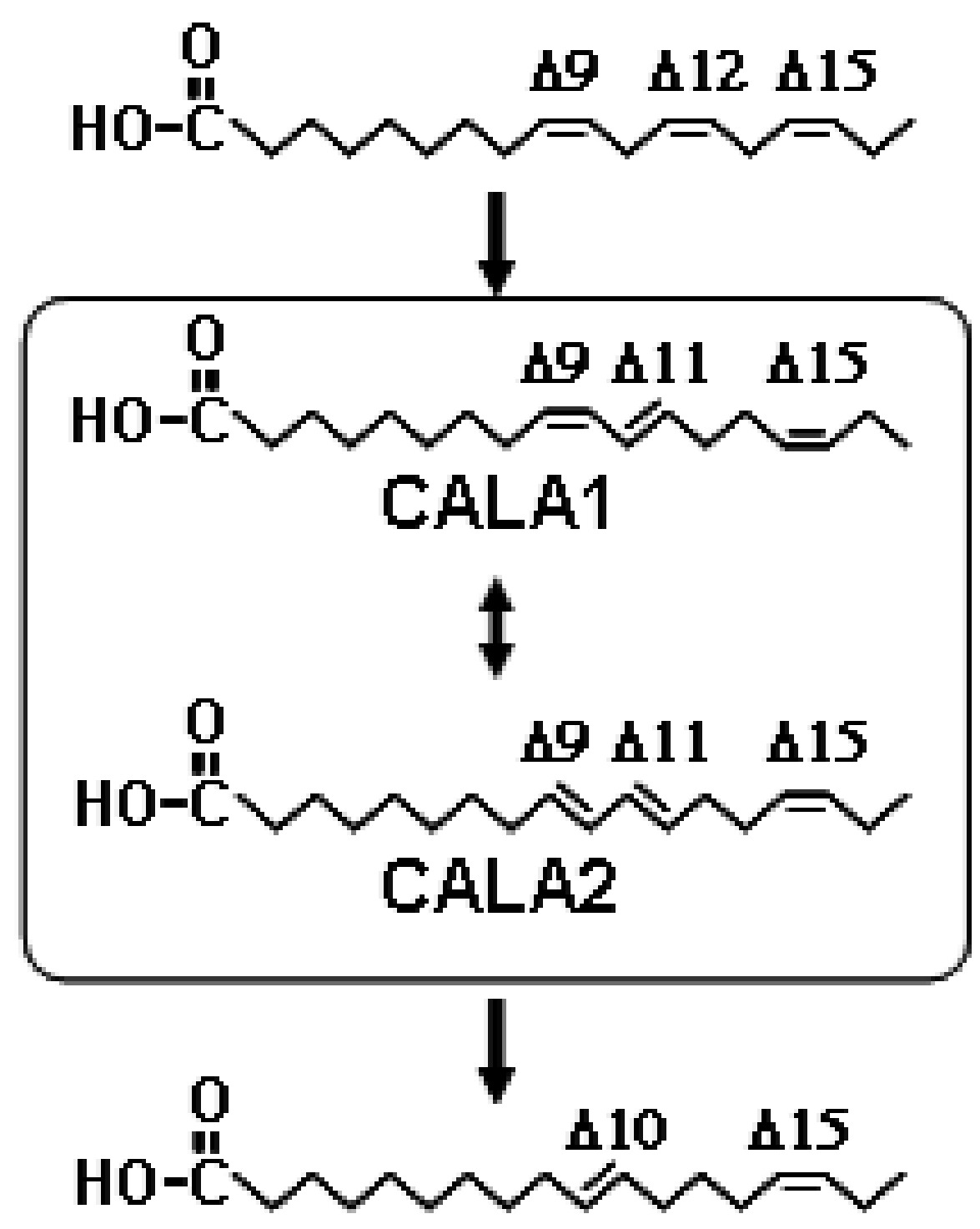

$y$-Linolenic acid

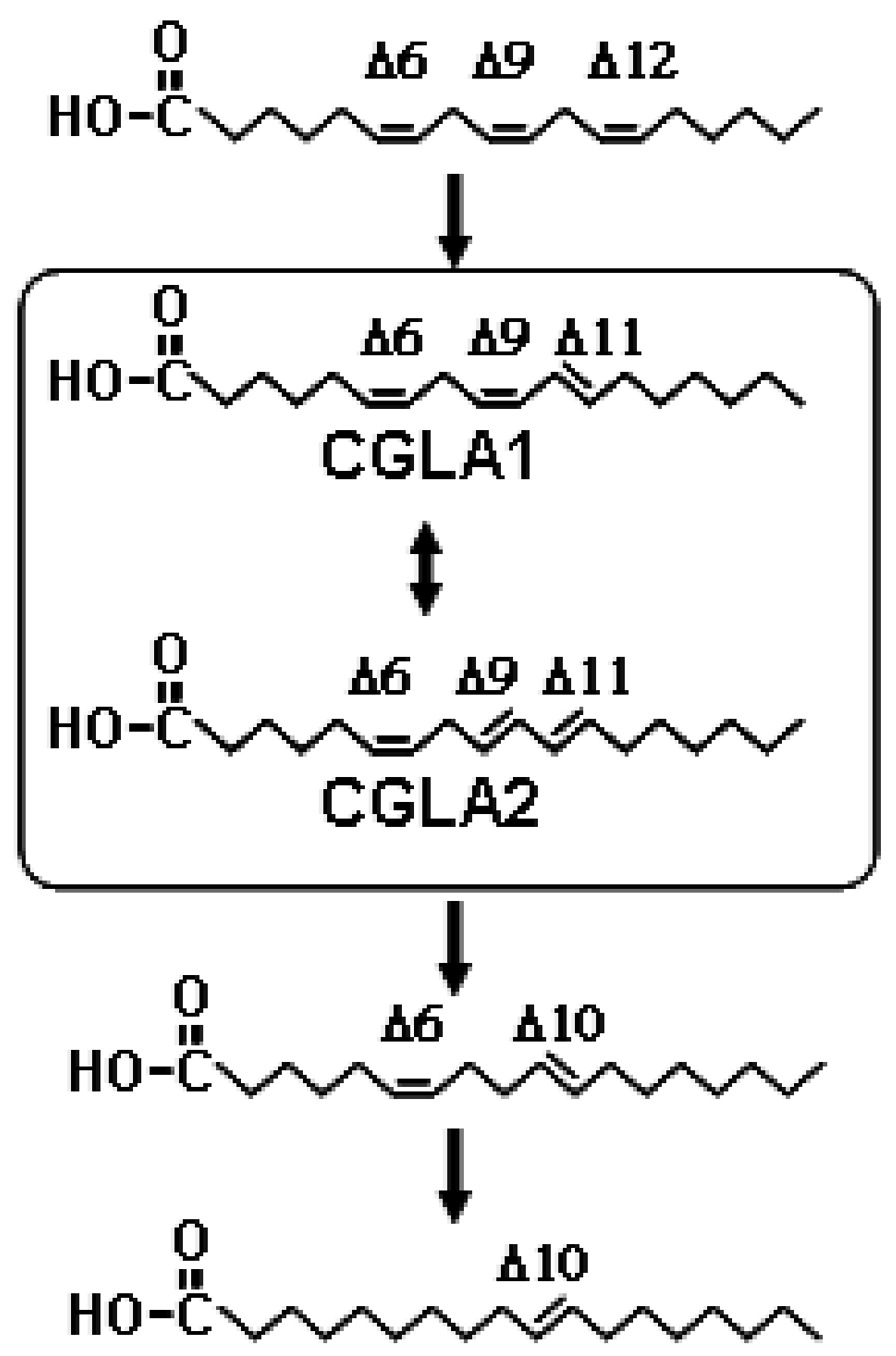




\section{Castor oil}

$$
1 \text { Lipase }
$$

\section{Ricin oleic acid}

[12-hydrong-cis-9-octadecenoio acid]

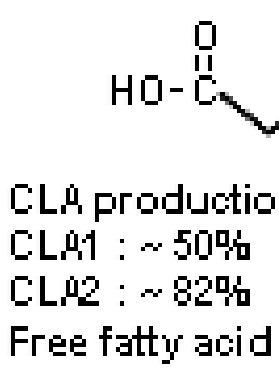

\section{Linoleic acid}

[ois-9,cis-12-ootadeosdienoic acid]

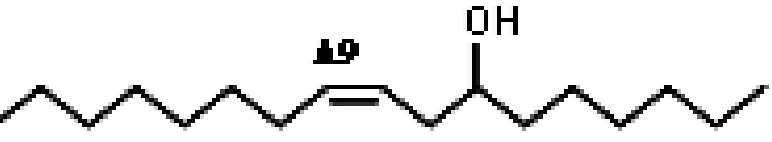

40

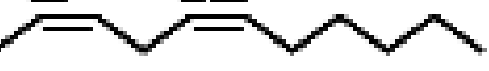

CLÁ production : $20040 \mathrm{mg} / \mathrm{ml}$ CLAB : -75 \%

CLAR: $: 97$ प'

Free fatty acid

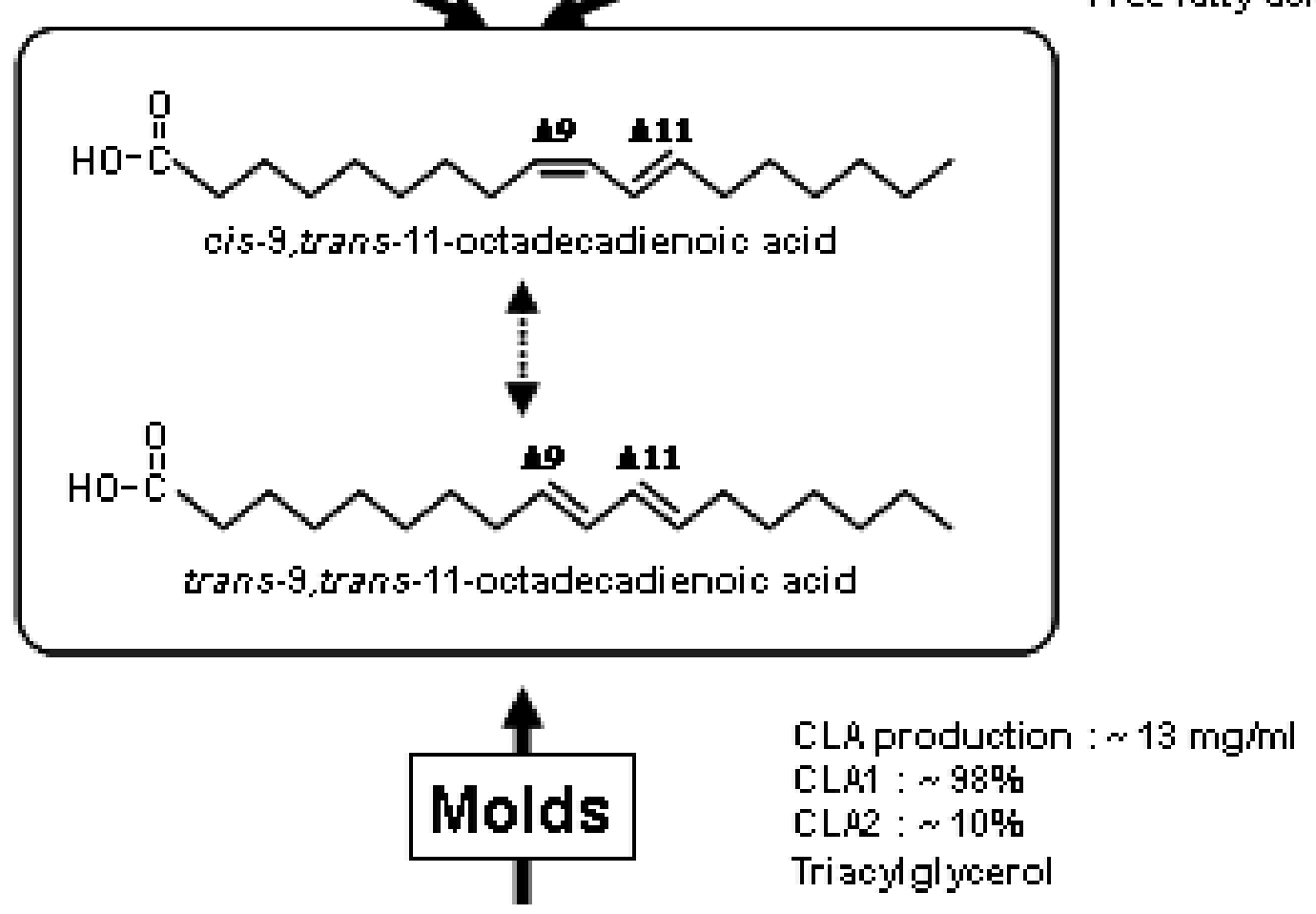

trans-Waccenic acid

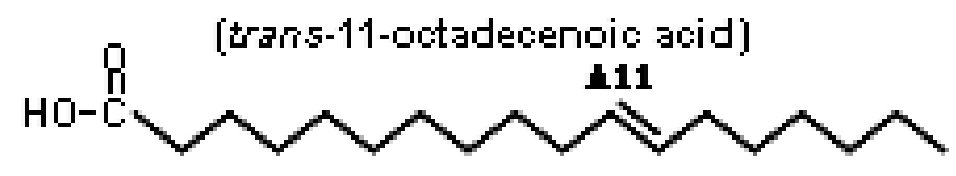

